\title{
Erratum to our paper On the divergence of Fourier series of functions in several variables DOI: $10.1007 / \mathrm{s} 10476-013-0301-1$
}

\author{
L. GOGOLADZE and V. TSAGAREISHVILI \\ I. Javakhishvili Tbilisi State University, University Str. 2, \\ 0186 Tbilisi, Georgia, e-mail: lgogoladze1@hotmail.com, cagare@ymail.com
}

Received October 16, 2015.

Abstract. We correct some errors and typos in our paper.

In our paper On the divergence of Fourier series of functions in several variables, Analysis Math., 39(2013), 163-178, we have noticed some errors after publication. The respective corrections are given below.

1. The last sentence of the abstract should read as: "For instance, for any $\varepsilon \in(0,2)$ and a given complete othonormal system there exists a function in $k>\frac{2(2-\varepsilon)}{\varepsilon}$ variables, having all the continuous partial derivatives of the first order, however, the series of absolute values of its coefficients with respect to the above system diverges in power $2-\varepsilon . "$

2. In the Russian summary the last sentence should read as: "Например, для любого $\varepsilon \in(0,2)$ и любой полной ортонормированной системы существует функция $k>\frac{2(2-\varepsilon)}{\varepsilon}$ переменных, имеющая все непрерывные частные производные первого порядка, однако ряд абсолютных значений ее коэффициентов относительно указанной системы расходится в степени $2-\varepsilon . "$

3. Theorem 5 should begin as: "Let $0<\varepsilon<2$ be an arbitrary number and let $\left(\varphi_{n}(x)\right)$ be a given complete ONS on $D_{k}$. Then there exists a 
function $f_{0}(X)$ of $k$ variables, $k \geq \frac{2(2-\varepsilon)}{\varepsilon}$, which has all the continuous partial derivatives of the first order, however, we have ..."

4. p. 164 (line 11 from below): the sign "-" is omitted.

5. p. 166 (line 6 from above): instead of $-h^{\alpha} h^{\alpha}$ there should be $-h^{\alpha}-h^{\alpha}$.

6. p. 168 (line 7 from above): $m$ and $m_{0}$ must be replaced by $n$ and $n_{0}$ and at the end of the line there must be $2^{k} 2^{-n_{0} \alpha}$.

7. p. 173 (the last line): in the upper index of the intersection instead of $m$ there should be $k$.

8. p. 175 (line 4 from above): instead of $m^{-1} n_{1}^{-1}$ there should be $m^{-1} \prod_{i=1}^{k} n_{i}^{-1}$.

9. p. 175 (line 7 from above): instead of $n_{i}^{-2}$ there should be $m^{-1} \prod_{i=1}^{k} n_{i}^{-2}$.

10. p. 175 (the last line): instead of $2^{-2 m}$ there should be $2^{-2 m k}$.

11. p. 177 (line 11 from above): instead of $B=\left\{f_{1}, f_{2}, \ldots, f_{l}\right\}$, $1 \leq l \leq k$ there should be $B=\left\{j_{1}, j_{2}, \ldots, j_{l}\right\}, 1 \leq l \leq k$.

12. p. 177 (line 12 from above): instead of $n_{j}$ there should be $n_{j_{i}}$. 